\title{
MIMO Radar Transmit Beampattern Design for DOA Estimation with Sidelobe Suppression
}

\author{
Yuxiang Li, ${ }^{1}$ Shuainan $\mathrm{Gu}^{2}$ and Nae Zheng ${ }^{1}$ \\ ${ }^{1}$ Zhengzhou Information Science and Technology Institute, Zhengzhou, Henan 86-450002, China \\ ${ }^{2}$ China National Digital Switching System Engineering and Technological Research Center, Zhengzhou, Henan 86-450002, China \\ Correspondence should be addressed to Yuxiang Li; liyuxiangwork@163.com
}

Received 27 October 2015; Accepted 14 January 2016

Academic Editor: Renato Cicchetti

Copyright (c) 2016 Yuxiang Li et al. This is an open access article distributed under the Creative Commons Attribution License, which permits unrestricted use, distribution, and reproduction in any medium, provided the original work is properly cited.

\begin{abstract}
We address the problem of transmit beamspace design for multiple-input multiple-output (MIMO) radar with colocated antennas in direction-of-arrival (DOA) estimation application. Three transmit beampattern sidelobe suppression strategies for designing the transmit beamspace matrix are introduced. The design of transmit beamspace matrix is based on minimizing the difference between a desired transmit beampattern and the actual one while keeping the sidelobe levels under control. Uniform elemental power distribution across the transmit antenna is guaranteed; at the same time, signal rotational invariance property is considered, which enables search-free based DOA estimation algorithms to be utilized at the receiver. The proposed optimization problems are nonconvex and are solved by using semidefinite programming relaxation technique. Moreover, the DOA estimation Cramer-Rao bound with transmit beamspace matrix is discussed. Simulation results show the superiority of the proposed techniques over the existing methods.
\end{abstract}

\section{Introduction}

The emerging concept of multiple-input multiple-output (MIMO) radar has been the focus of intensive research recently [1]. Depending on the array element configuration, MIMO radar can be classified into two categories: widely distributed $[2-4]$ and colocated $[5,6]$. In the widely distributed case, the transmitting antennas are widely separated so that each antenna may view a different aspect of the target, which can increase the spatial diversity of the system. In colocated systems, the transmitting antennas are closely spaced to view the same aspect of the target, which can increase the spatial resolution of the system [5]. Compared to phased-array radar, MIMO radar enjoys the advantage of waveform diversity but has drawbacks in terms of signal-to-ratio (SNR) loss. To preserve the waveform diversity of MIMO radar while taking the advantage of the coherent processing gain of phasedarray radar, new configuration of radar architecture such as the phased-MIMO radar has been proposed $[7,8]$. The ideas discussed in these works are transmitting multiple beams from subarrays, each of which is generated by using a different waveform.
Besides these array partitioning based techniques, several transmit beamforming methods have been developed in the literature to achieve transmit coherent gain in MIMO radar under the assumption that the targets are located within an already known spatial sector. The design of waveforms to achieve desired beampattern is usually a two-step process. In the first step, a covariance matrix of the waveforms is synthesized to obtain the desired beampattern $[9,10]$. In the second step, the actual waveforms are designed to realize the synthesized covariance matrix while keeping the designed waveforms having low peak-to-average power ratios (PAPRs) or having constant envelope. Note that these methods are challenging and computationally demanding $[11,12]$.

To reduce the computational burden of the two-step process, a more efficient technique called transmit beamspace processing (TBP) was proposed in [13]. The TBP technique introduces a beamspace weighting matrix to several orthogonal waveforms used in the standard MIMO radar. In this way, the DOA estimation performance can be improved due to the concentration of transmit power in the desired spatial sector, and it is also possible to guarantee the rotational invariance property (RIP) at the receive array. However, [13] suffers 
from the shortcoming that the transmit power distribution across the antenna array elements is not uniform. In [14], two kinds of weighting matrices are proposed which can be easily optimized for desired beampattern by using hypersphere coordinates transformation technique. The drawback of [14] is that the resulting PAPR is a little great. In [15], the TBP based beampattern design problem is reformulated and a spatial-division based design method is also proposed, resulting in DOA estimation improvement.

Although many solutions and discussions have been made upon this problem, very little attention has been paid to the sidelobe suppression issue in transmit beamspace processing. In the design of transmit beampattern, sidelobe suppression operation can reduce the transmit power loss in the uninterested regions, which leads to higher SNR at the receive antennas, and then a better DOA estimation performance can be achieved potentially. In this paper, a multiaspect discussion of transmit beamspace design with sidelobe suppression operations for DOA estimation is provided, and the major contributions of this paper are noted below:

(1) In the proposed scheme, sidelobe levels can be restrained by using three different criteria. (a) Minimize the error between the designed beampattern and the desired one in the main-lobe region, and simultaneously guarantee that the sidelobe levels are lower than a given threshold. (b) Minimize the sidelobe levels, and at the same time guarantee that the error between the designed beampattern and the desired one is smaller than a certain value. (c) Minimize the sidelobes, while keeping the main-beam pointing toward a desired direction.

(2) In the proposed sidelobe suppression strategies, we reformulate the nonconvex transmit beampattern design problem as convex problems by means of semidefinite programming relaxation (SDP) technique. Then, the problems can be efficiently solved by using interior point method.

(3) To allow for simple search-free DOA estimation algorithms, the RIP at the receiver is guaranteed by imposing a specific structure on the transmit matrix. Moreover, the DOA estimation Cramer-Rao bound (CRB) with transmit beamspace matrix is analyzed.

This paper is organized as follows. Section 2 introduces MIMO radar signal model with transmit beamspace matrix. In Section 3, we propose three sidelobe suppression strategies in the design of transmit beampattern. Section 4 provides the performance analysis using numerical examples. The conclusions are drawn in Section 5.

\section{MIMO Radar Signal Model}

Consider a monostatic MIMO radar system equipped with a transmit array of $M$ collocated antennas with interelement spacing $d_{t}$ and a receive array of $N$ collocated antennas with interelement spacing $d_{r}$. The transmit and receive arrays are assumed to be close enough to each other such that the spatial angle of a target in the far-field remains the same with respect to transmit and receive arrays.

Instead of transmitting omnidirectionally as the traditional MIMO radar does, we intend to focus the transmitted energy within an interested sector $\Theta$ by introducing a transmit beamspace weighting matrix $\mathbf{W}$. Therefore, the actual transmitted signals are taken as linear combinations of $K$ traditional orthogonal waveforms $\varphi(t)=\left[\varphi_{1}(t), \ldots, \varphi_{K}(t)\right]^{T}$, $(K \leq M)$. The baseband representation of the transmitted signals can be written as $\mathbf{s}(t)=\left[s_{1}(t), \ldots, s_{M}(t)\right]^{T}=\mathbf{W}^{*} \boldsymbol{\varphi}(t)$, where $\mathbf{W}=\left[\mathbf{w}_{1}, \ldots, \mathbf{w}_{K}\right]$ is the $M \times K$ transmit beamspace matrix, where $\mathbf{w}_{k}$ is the $k$ th unit-form weight vector. It is assumed that $\int_{0}^{T} \varphi_{i}(t) \varphi_{j}^{*}(t)=\delta(i-j), i, j=1, \ldots, K$, where $T$ is the pulse duration, $(\cdot)^{T}$ and $(\cdot)^{*}$ stand for the transpose and the conjugate, respectively, and $\delta(\cdot)$ denotes the Kronecker delta.

By using TBP model, the received signal at location $\theta$ can be written as [13]

$$
\mathbf{x}(t, \theta)=\sum_{k=1}^{K} \mathbf{a}^{T}(\theta) \mathbf{w}_{k}^{*} \varphi_{k}(t)=\left(\mathbf{W}^{H} \mathbf{a}(\theta)\right)^{T} \boldsymbol{\varphi}(t),
$$

where $\mathbf{a}(\theta)$ is the $M \times 1$ transmit array steering vector and $(\cdot)^{H}$ denotes the conjugate transpose. Assume that $P$ targets are present in the space. The reflection coefficient of each target is assumed to be constant during the whole pulse but varies independently from pulse to pulse; that is, they obey the Swerling II target model. Therefore, receive array observations can be written as

$$
\begin{aligned}
\mathbf{r}(t, \tau)= & \sum_{p=1}^{P} \beta_{p}(\tau)\left(\mathbf{W}^{H} \mathbf{a}\left(\theta_{p}\right)\right)^{T} \boldsymbol{\varphi}(t) \mathbf{b}\left(\theta_{p}\right) \\
& +\mathbf{n}(t, \tau)
\end{aligned}
$$

where $t$ is the time index within the radar pulse, $\tau$ is the slow time index, that is, the pulse number, $\beta_{p}(\tau)$ is the reflection coefficient, $\mathbf{b}\left(\theta_{p}\right)$ is steering vector of the receive array associated with the $p$ th target, and $\mathbf{n}(t, \tau)$ is the $N \times 1$ vector of zero-mean white Gaussian noise.

Exploiting the orthogonal property of the traditional orthogonal waveforms, the components of received data can be separated by using matched filtering technique. By stacking the individual vector components in one column vector, we can obtain the entire virtual receive data vector [15]

$$
\mathbf{y}(\tau)=\sum_{p=1}^{P} \beta_{p}(\tau)\left(\mathbf{W}^{H} \mathbf{a}\left(\theta_{p}\right)\right) \otimes \mathbf{b}\left(\theta_{p}\right)+\widetilde{\mathbf{n}}(\tau),
$$

where $\otimes$ denotes the Kronecker product, $\widetilde{\mathbf{n}}(\tau)=$ $\left[\mathbf{n}_{1}^{T}(\tau), \ldots, \mathbf{n}_{K}^{T}(\tau)\right]$ is the $K N \times 1$ Gaussian noise term whose covariance is given by $\sigma_{n}^{2} \mathbf{I}_{K N}$, and $\mathbf{I}_{K N}$ is the identity matrix of size $K N \times K N$.

\section{Problem Formulation}

The main goal of transmit beampattern design is to devise a transmit beamspace matrix $\mathbf{W}$ which achieves a transmit 
beampattern that is as close as possible to the desired one. Actually, the transmit beampattern at spatial angle $\theta$ can be defined as [9]

$$
\begin{aligned}
P(\theta) & =\mathbf{a}^{H}(\theta) E\left\{\mathbf{s}(t) \mathbf{s}^{H}(t)\right\} \mathbf{a}(\theta)=\left\|\mathbf{W}^{H} \mathbf{a}(\theta)\right\|^{2} \\
& =\sum_{k=1}^{K} \mathbf{w}_{k}^{H} \mathbf{a}(\theta) \mathbf{a}^{H}(\theta) \mathbf{w}_{k} .
\end{aligned}
$$

Let $P_{d}\left(\theta_{l}\right)$ denote the desired beampattern value at the location of $\theta_{l}$; then the transmit beamspace matrix design problem can be formulated as

$$
\min \left\{\sum_{l=1}^{L}\left|\sum_{k=1}^{K} \mathbf{w}_{k}^{H} \mathbf{a}(\theta) \mathbf{a}^{H}(\theta) \mathbf{w}_{k}-\alpha P_{d}\left(\theta_{l}\right)\right|^{p}\right\},
$$

where $\alpha$ is an optimal scaling factor and $p=1,2$, or $\infty$ denotes the $l_{1}$-norm, $l_{2}$-norm, and $l_{\infty}$-norm, respectively.

Next, we want to transmit the same power from each antenna. Let $P_{t}$ be the total transmit power; we incorporate the uniform power distribution across transmit array antenna elements by using the following constraints:

$$
\sum_{k=1}^{K}\left|\mathbf{w}_{k}(j)\right|^{2}=\frac{P_{t}}{M}, \quad j=1, \ldots, M
$$

In practice, each antenna in the transmit array usually uses the same power amplifier and thus has the same dynamic power range. If the power used by different antennas is allowed to vary widely, this can severely degrade the performance of the radar system due to the nonlinear characteristics of the power amplifier.

3.1. Transmit Beampattern Design with Sidelobe Suppression. By reducing transmit power in the sidelobe regions, the amount of energy that otherwise could be wasted in the undesired areas can be added to the amount of energy to be transmitted within the desired spatial sector. As a result, the signal strength within the desired sector can be increased, which potentially leads to better achievable DOA estimation performance. In this subsection, let $\theta_{l} \in \Theta, l=1, \ldots, L$, and $\theta_{s} \in \bar{\Theta}, s=1, \ldots, S$, be a uniform grid of points that cover the main-lobe regions and sidelobe regions, respectively. Three sidelobe suppression strategies for designing the transmit beamspace matrix $\mathbf{W}$ are proposed.

Strategy 1 . The criterion is to minimize the $l_{2}$-norm difference between the desired beampattern and the actual beampattern, while maintaining sidelobes to be lower than given threshold $\eta$ and at the same time ensuring equal transmit power at each antenna. This strategy operates sidelobe restriction directly by setting a threshold on the sidelobe level, which can be usually applied to the scenario where small sidelobe fluctuation is required. Mathematically, this kind of strategy can be expressed as

$$
\begin{array}{ll}
\min _{\alpha, \mathbf{w}} & \mathbf{J}_{1}=\sum_{l=1}^{L}\left|\sum_{k=1}^{K} \mathbf{w}_{k}^{H} \mathbf{a}\left(\theta_{l}\right) \mathbf{a}^{H}\left(\theta_{l}\right) \mathbf{w}_{k}-\alpha P_{d}\left(\theta_{l}\right)\right|^{2} \\
\text { s.t. } & \left|\sum_{k=1}^{K} \mathbf{w}_{k}^{H} \mathbf{a}\left(\theta_{s}\right) \mathbf{a}^{H}\left(\theta_{s}\right) \mathbf{w}_{k}\right| \leq \eta, \\
& \theta_{s} \in \bar{\Theta}, s=1, \ldots, S \\
& \sum_{k=1}^{K}\left|\mathbf{w}_{k}(j)\right|^{2}=\frac{P_{t}}{M}, \quad j=1, \ldots, M .
\end{array}
$$

It should be noted that problem (7) belongs to the category of nonconvex quadratically constrained quadratic programming problems which are in general NP-hard. However, semidefinite programming relaxation technique [16] can be used to approximately solve it. Introducing the auxiliary variables $\mathbf{X}_{k}=\mathbf{w}_{k} \mathbf{w}_{k}^{H},(k=1, \ldots, K)$, and according to the properties $\mathbf{w}_{k}^{H} \mathbf{a}\left(\theta_{l}\right) \mathbf{a}^{H}\left(\theta_{l}\right) \mathbf{w}_{k}=\operatorname{tr}\left\{\mathbf{a}\left(\theta_{l}\right) \mathbf{a}^{H}\left(\theta_{l}\right) \mathbf{w}_{k} \mathbf{w}_{k}^{H}\right\}$, the problem can be equivalently written as

$$
\begin{aligned}
& \min _{\alpha, \mathbf{X}_{k}} \sum_{l=1}^{L}\left|\sum_{k=1}^{K} \operatorname{tr}\left\{\mathbf{a}\left(\theta_{l}\right) \mathbf{a}^{H}\left(\theta_{l}\right) \mathbf{X}_{k}\right\}-\alpha P_{d}\left(\theta_{l}\right)\right|^{2} \\
& \text { s.t. }\left|\sum_{k=1}^{K} \operatorname{tr}\left\{\mathbf{a}\left(\theta_{s}\right) \mathbf{a}^{H}\left(\theta_{s}\right) \mathbf{X}_{k}\right\}\right| \leq \eta, \\
& \qquad \theta_{s} \in \bar{\Theta}, s=1, \ldots, S \\
& \\
& \sum_{k=1}^{K} \operatorname{diag}\left\{\mathbf{X}_{k}\right\}=\frac{P_{t}}{M} \mathbf{1}_{M \times 1} \\
& \operatorname{rank}\left(\mathbf{X}_{k}\right)=1, \quad k=1, \ldots, K,
\end{aligned}
$$

where $\operatorname{tr}(\cdot)$ stands for the $\operatorname{trace}, \operatorname{diag}(\cdot)$ denotes the diagonal of a square matrix, and $\operatorname{rank}(\cdot)$ denotes the rank of a matrix. The optimization problem (8) remains nonconvex due to the fact that the rank constraint $\operatorname{rank}\left(\mathbf{X}_{k}\right)=1$ is nonconvex. Therefore, by means of SDP relaxation technique, we can relax the rank constraint from $\operatorname{rank}\left(\mathbf{X}_{k}\right)=1$ to the constraint that $\mathbf{X}_{k}$ is positively semidefinite; that is, $\mathbf{X}_{k} \geq 0$. By introducing new variables $\left\{\delta_{l}\right\}_{l=1}^{L}\left(\delta_{l}>0, l=1, \ldots, L\right)$, problem (8) can be represented as a convex one

$$
\begin{aligned}
& \min _{\alpha, \mathbf{X}_{k}} \sum_{l=1}^{L} \delta_{l} \\
& \text { s.t. }\left|\sum_{k=1}^{K} \operatorname{tr}\left\{\mathbf{a}\left(\theta_{l}\right) \mathbf{a}^{H}\left(\theta_{l}\right) \mathbf{X}_{k}\right\}-\alpha P_{d}\left(\theta_{l}\right)\right|^{2} \leq \delta_{l}, \\
& \theta_{l} \in \Theta, l=1, \ldots, L \\
&\left|\sum_{k=1}^{K} \operatorname{tr}\left\{\mathbf{a}\left(\theta_{s}\right) \mathbf{a}^{H}\left(\theta_{s}\right) \mathbf{X}_{k}\right\}\right| \leq \eta, \\
& \theta_{s} \in \bar{\Theta}, s=1, \ldots, S
\end{aligned}
$$




$$
\begin{aligned}
& \sum_{k=1}^{K} \operatorname{diag}\left\{\mathbf{X}_{k}\right\}=\frac{P_{t}}{M} \mathbf{1}_{M \times 1} \\
& \mathbf{X}_{k} \geq 0, \quad k=1, \ldots, K .
\end{aligned}
$$

Note that the quadratic inequality constraint in the above problem can be rewritten as

$$
\begin{aligned}
& \left|\sum_{k=1}^{K} \operatorname{tr}\left\{\mathbf{a}\left(\theta_{l}\right) \mathbf{a}^{H}\left(\theta_{l}\right) \mathbf{X}_{k}\right\}-\alpha P_{d}\left(\theta_{l}\right)\right|^{2} \leq \delta_{l} \Longleftrightarrow \\
& \left|\sum_{k=1}^{K} 2 \cdot \operatorname{tr}\left\{\mathbf{a}\left(\theta_{l}\right) \mathbf{a}^{H}\left(\theta_{l}\right) \mathbf{X}_{k}\right\}-2 \alpha P_{d}\left(\theta_{l}\right)\right|^{2}+\delta_{l}^{2}-2 \delta_{l} \\
& \quad+1 \leq \delta_{l}^{2}+2 \delta_{l}+1 .
\end{aligned}
$$

Using standard properties of matrix norm, the quadratic inequality can be expressed as a second-order cone in the following form:

$$
\left.\begin{array}{c}
\left\|\sum_{k=1}^{K} 2 \cdot \operatorname{tr}\left\{\mathbf{a}\left(\theta_{l}\right) \mathbf{a}^{H}\left(\theta_{l}\right) \mathbf{X}_{k}\right\}-2 \alpha P_{d}\left(\theta_{l}\right)\right\|^{2} \leq\left(\delta_{l}+1\right)^{2} \Longleftrightarrow \\
\delta_{l}-1
\end{array}\right)
$$

where $\|\cdot\|$ denotes the Frobenius matrix norm. Therefore, problem (9) can be formulated as the following second-order cone programming (SOCP) problem:

$$
\begin{array}{ll}
\min _{\alpha, \mathbf{X}_{k}} & \sum_{l=1}^{L} \delta_{l} \\
\text { s.t. } & \mid \sum_{k=1}^{K} 2 \cdot \operatorname{tr}\left\{\mathbf{a}\left(\theta_{l}\right) \mathbf{a}^{H}\left(\theta_{l}\right) \mathbf{X}_{k}\right\}-2 \alpha P_{d}\left(\theta_{l}\right) \| \\
& \leq\left(\delta_{l}+1\right), \quad \delta_{l}-1 \\
& \left|\sum_{k=1}^{K} \operatorname{tr}\left\{\mathbf{a}\left(\theta_{s}\right) \mathbf{a}^{H}\left(\theta_{s}\right) \mathbf{X}_{k}\right\}\right| \leq \eta, l=1, \ldots, L \\
& \sum_{k=1}^{K} \operatorname{diag}\left\{\mathbf{X}_{k}\right\}=\frac{P_{t}}{M} \mathbf{1}_{M \times 1} \\
& \mathbf{X}_{k} \geq 0, \quad k=1, \ldots, K .
\end{array}
$$

Note that this SOCP problem can be efficiently solved using well-established interior point methods software tools. Specifically, the CVX MATLAB toolbox [17] is used in this paper.

Strategy 2. The goal of this strategy is to minimize the peak sidelobe level by using minimax criterion, as well as keeping the difference between the desired beampattern and the actual one below a certain level $\xi$, and ensure equal transmit power at each antenna. Such a strategy can be formulated as

$$
\begin{aligned}
\min _{\alpha, \mathbf{w}} \max _{\theta_{s}} & \mathbf{J}_{2}=\left|\sum_{k=1}^{K} \mathbf{w}_{k}^{H} \mathbf{a}\left(\theta_{s}\right) \mathbf{a}^{H}\left(\theta_{s}\right) \mathbf{w}_{k}\right| \\
\text { s.t. } & \sum_{l=1}^{L}\left|\sum_{k=1}^{K} \mathbf{w}_{k}^{H} \mathbf{a}\left(\theta_{l}\right) \mathbf{a}^{H}\left(\theta_{l}\right) \mathbf{w}_{k}-\alpha P_{d}\left(\theta_{l}\right)\right| \leq \xi, \\
& \theta_{l} \in \Theta, l=1, \ldots, L \\
& \sum_{k=1}^{K}\left|\mathbf{w}_{k}(j)\right|^{2}=\frac{P_{t}}{M}, \quad j=1, \ldots, M,
\end{aligned}
$$

where $\xi$ is a given difference upper bound between the desired beampattern and the actual one in $l_{1}$-norm. Note that Strategy 2 uses an opposite way to restrict sidelobes compared with Strategy 1 which directly imposes a threshold on the sidelobe level. Because this optimization problem is nonconvex, in a similar way, we substitute variable $\mathbf{X}_{k}=\mathbf{w}_{k} \mathbf{w}_{k}^{H}$ in (13) and utilize the SDP relaxation technique, and then the problem can be rewritten as

$$
\begin{aligned}
\min _{\alpha, \mathbf{X}} \max _{\theta_{s}} & \left|\sum_{k=1}^{K} \operatorname{tr}\left\{\mathbf{a}\left(\theta_{s}\right) \mathbf{a}^{H}\left(\theta_{s}\right) \mathbf{X}_{k}\right\}\right| \\
\text { s.t. } & \sum_{l=1}^{L}\left|\sum_{k=1}^{K} \operatorname{tr}\left\{\mathbf{a}\left(\theta_{l}\right) \mathbf{a}^{H}\left(\theta_{l}\right) \mathbf{X}_{k}-\alpha P_{d}\left(\theta_{l}\right)\right\}\right| \\
& \leq \xi, \\
& \sum_{k=1}^{K} \operatorname{diag}\left\{\mathbf{X}_{k}\right\}=\frac{P_{t}}{M} \mathbf{1}_{M \times 1}, l=1, \ldots, L \\
& \mathbf{X}_{k} \geq 0, \quad k=1, \ldots, K .
\end{aligned}
$$

After introducing a new auxiliary variable $\delta(\delta>0)$, problem (14) can be equivalently expressed as

$$
\begin{array}{ll}
\min _{\alpha, \mathbf{X}} & \delta \\
\text { s.t. } & \left|\sum_{k=1}^{K} \operatorname{tr}\left\{\mathbf{a}\left(\theta_{s}\right) \mathbf{a}^{H}\left(\theta_{s}\right) \mathbf{X}_{k}\right\}\right| \leq \delta, \\
& \theta_{s} \in \bar{\Theta}, s=1, \ldots, S \\
& \sum_{l=1}^{L}\left|\sum_{k=1}^{K} \operatorname{tr}\left\{\mathbf{a}\left(\theta_{l}\right) \mathbf{a}^{H}\left(\theta_{l}\right) \mathbf{X}-\alpha P_{d}\left(\theta_{l}\right)\right\}\right| \leq \xi, \\
& \sum_{k=1}^{K} \operatorname{diag}\left\{\mathbf{X}_{k}\right\}=\frac{P_{t}}{M} \mathbf{1}_{M \times 1} \\
& \mathbf{X}_{k} \geq 0, \quad k=1, \ldots, K .
\end{array}
$$


In a similar way, problem (15) can be efficiently solved via the CVX toolbox.

Strategy 3. Motivated by the sidelobe suppression method for traditional beampattern design proposed in [18], we extend the idea into the TBP model. The criterion is to maximize the difference between the main-beam and the peak sidelobe level under the constraint that the $3 \mathrm{~dB}$ width of the mainbeam is given. This strategy can be formulated as follows:

$$
\begin{aligned}
\min _{\mathbf{X}} \max _{\theta_{s}} & \mathbf{J}_{3}=-\left|\sum_{k=1}^{K} \mathbf{w}_{k}^{H} \mathbf{a}\left(\theta_{0}\right) \mathbf{a}^{H}\left(\theta_{0}\right) \mathbf{w}_{k}-\sum_{k=1}^{K} \mathbf{w}_{k}^{H} \mathbf{a}\left(\theta_{s}\right) \mathbf{a}^{H}\left(\theta_{s}\right) \mathbf{w}_{k}\right| \\
\text { s.t. } & \sum_{k=1}^{K} \mathbf{w}_{k}^{H} \mathbf{a}\left(\theta_{1}\right) \mathbf{a}^{H}\left(\theta_{1}\right) \mathbf{w}_{k}=0.5 \sum_{k=1}^{K} \mathbf{w}_{k}^{H} \mathbf{a}\left(\theta_{0}\right) \mathbf{a}^{H}\left(\theta_{0}\right) \mathbf{w}_{k} \\
& \sum_{k=1}^{K} \mathbf{w}_{k}^{H} \mathbf{a}\left(\theta_{2}\right) \mathbf{a}^{H}\left(\theta_{2}\right) \mathbf{w}_{k}=0.5 \sum_{k=1}^{K} \mathbf{w}_{k}^{H} \mathbf{a}\left(\theta_{0}\right) \mathbf{a}^{H}\left(\theta_{0}\right) \mathbf{w}_{k} \\
& \sum_{k=1}^{K}\left|\mathbf{w}_{k}(j)\right|^{2}=\frac{P_{t}}{M}, \quad j=1, \ldots, M,
\end{aligned}
$$

where $\theta_{0}$ denotes the direction of the main-beam and $\theta_{2}-$ $\theta_{1}\left(\theta_{0}>\theta_{1}, \theta_{2}>\theta_{0}\right)$ determines the $3 \mathrm{~dB}$ main-beam width. In a similar way, by introducing a new auxiliary variable $\sigma$ $(\sigma>0)$ and utilizing the SDP relaxation technique, problem (16) can be equivalently expressed as

$$
\begin{array}{ll}
\min _{\mathbf{X}} & -\sigma \\
\text { s.t. } & \sum_{k=1}^{K} \operatorname{tr}\left\{\mathbf{a}\left(\theta_{0}\right) \mathbf{a}^{H}\left(\theta_{0}\right) \mathbf{X}_{k}\right\} \\
& -\sum_{k=1}^{K} \operatorname{tr}\left\{\mathbf{a}\left(\theta_{s}\right) \mathbf{a}^{H}\left(\theta_{s}\right) \mathbf{X}_{k}\right\} \geq \sigma, \\
& \sum_{k=1}^{K} \operatorname{tr}\left\{\mathbf{a}\left(\theta_{1}\right) \mathbf{a}^{H}\left(\theta_{1}\right) \mathbf{X}_{k}\right\} \\
& =0.5 \sum_{k=1}^{K} \operatorname{tr}\left\{\mathbf{a}\left(\theta_{0}\right) \mathbf{a}^{H}\left(\theta_{0}\right) \mathbf{X}_{k}\right\} \\
& \sum_{k=1}^{K} \operatorname{tr}\left\{\mathbf{a}\left(\theta_{2}\right) \mathbf{a}^{H}\left(\theta_{2}\right) \mathbf{X}_{k}\right\} \\
& \sum_{k=1}^{K} \operatorname{diag}\left\{\mathbf{X}_{k}\right\}=0, \quad k=1, \ldots, K . \\
& =0.5 \sum_{k=1}^{K} \operatorname{tr}\left\{\mathbf{a}\left(\theta_{0}\right) \mathbf{a}^{H}\left(\theta_{0}\right) \mathbf{X}_{k}\right\} \\
& \\
& \\
& \\
& \\
& \\
&
\end{array}
$$

Apparently, problem (17) is convex, which can be efficiently solved via optimization toolbox CVX.
3.2. Enforcing the RIP and Solving the Beamspace Matrix. We consider to ensure that the received signal holds the rotational invariance property. RIP can enable us to use search-free ESPRIT-based DOA estimation techniques, which require small computational cost compared with MUSIC-based DOA estimation techniques. Enforcing the RIP is equivalent to ensuring that the following relationship holds [15]:

$$
\begin{aligned}
\left|\mathbf{a}(\theta)^{H} \mathbf{w}_{k}\right|=\left|\mathbf{a}(\theta)^{H} \mathbf{w}_{K / 2+k}\right|, & \\
\theta & \in\left[-\frac{\pi}{2}, \frac{\pi}{2}\right], k=1, \ldots, \frac{K}{2},
\end{aligned}
$$

where $K$ is assumed to be an even number. Under the assumption of ULA at the MIMO radar transmitter, the RIP can be satisfied by choosing the transmit beamspace matrix to take the form

$$
\mathbf{W}=\left[\mathbf{w}_{1}, \ldots, \mathbf{w}_{K / 2}, \widetilde{\mathbf{w}}_{1}^{*}, \ldots, \widetilde{\mathbf{w}}_{K / 2}^{*}\right]
$$

where $\widetilde{\mathbf{w}}$ is the flipped version of vector $\mathbf{w}$; that is, $\widetilde{w}_{k}(i)=$ $w_{k}(M-i+1), i=1, \ldots, M$. We consider $K=2$ as a special case to explain the reason that $\mathbf{W}$ in (18) ensures the RIP. Actually, when $K=2$, the transmit beamspace matrix takes the form $\mathbf{W}=\left[\mathbf{w}, \widetilde{\mathbf{w}}^{*}\right]$, and thus the following relationship holds:

$$
\begin{gathered}
\mathbf{a}(\theta)^{H} \mathbf{w}=\sum_{k=1}^{M} w_{k} e^{-j 2 \pi \sin (\theta)(k-1)}, \\
\mathbf{a}(\theta)^{H} \widetilde{\mathbf{w}}^{*}=\sum_{k=1}^{M} w_{k}^{*} e^{-j 2 \pi \sin (\theta)(M-k)} .
\end{gathered}
$$


By factoring out the term $e^{-j 2 \pi \sin (\theta)(M-k)}$ from the right hand side of (20) and conjugating it, (21) can be equivalently rewritten as

$$
\begin{aligned}
\mathbf{a}(\theta)^{H} \widetilde{\mathbf{w}}^{*} & =\left(\sum_{k=1}^{M} w_{k} e^{-j 2 \pi \sin (\theta)(k-1)}\right)^{*} e^{-j 2 \pi \sin (\theta)(M-k)} \\
& =\left(\mathbf{a}(\theta)^{H} \mathbf{w}\right)^{*} e^{-j 2 \pi \sin (\theta)(M-1)} .
\end{aligned}
$$

From (22), it can be seen that the terms $\mathbf{a}(\theta)^{H} \mathbf{w}$ and $\mathbf{a}(\theta){ }^{H} \widetilde{\mathbf{w}}^{*}$ are identical in magnitude; therefore, the relationship in (18) is satisfied, that is, the RIP is ensured. For the case that $K>2$, the RIP can be demonstrated to be held in the same way.

After obtaining the solutions of the three relaxed versions of problems (7), (13), and (16) via CVX in the previous section, then we consider extracting the original solutions of them via the so-called Gaussian randomization technique [19]. Note that we can obtain the transmit beamspace matrix if only the terms $\mathbf{w}_{1}, \ldots, \mathbf{w}_{K / 2}$ are resolved. The algorithm to solve the transmit beamspace matrix is summarized in the following part.

\section{The Gaussian Randomization Algorithm to Solve the Transmit Beamspace Matrix}

Step 0 . Set the number of randomization, $N_{g}$. Let $\mathbf{X}_{k}^{\text {opt }}$, $(k=1, \ldots, K / 2)$, denote the optimal solution of the relaxed problem.

Step 1. If the rank of $\mathbf{X}_{\text {opt }}$ equals one, the optimal solution of $\mathbf{w}_{k}$ can be obtained by simply finding the principal eigenvector of $\mathbf{X}_{k}^{\text {opt }}$; else if the rank of matrix $\mathbf{X}_{k}^{\text {opt }}$ is higher than one, let $\mathbf{X}_{k}^{\text {opt }}=\mathbf{U}_{k} \boldsymbol{\Sigma}_{k} \mathbf{U}_{k}^{H}$ denote the eigendecomposition of $\mathbf{X}_{k}^{\text {opt }}$, where $\mathbf{U}_{k}$ and $\boldsymbol{\Sigma}_{k}$ are the matrix of eigenvectors and the diagonal matrix of the eigenvalues of $\mathbf{X}_{k}^{\text {opt }}$, respectively. The candidate vector $\mathbf{w}_{k}^{l}$ can be chosen as $\mathbf{w}_{k}^{l}=\mathbf{U}_{k} \Sigma_{k}^{1 / 2} \mathbf{v}_{k}^{l}$, where $v_{k}^{l}\left(l=1, \ldots, N_{g}\right)$ is a random vector whose elements are random variables uniformly distributed on the unit circle in the complex plane.

Step 2. If the constraint that each element of the vector $\sum_{k=1}^{K} \operatorname{diag}\left\{\mathbf{w}_{k}^{l}\left(\mathbf{w}_{k}^{l}\right)^{H}\right\}$ equals $P_{t} / M \times \mathbf{1}_{M \times 1}$ does not hold, we map the resulting random vectors to a nearby feasible point $\mathbf{w}_{k \text {,new }}^{l}$ by scaling the elements of each candidate vector so that the aforementioned constraint is satisfied.

Step 3. For Strategy 1, calculate the objective function $\mathbf{J}_{1}$ in (7). For Strategy 2, calculate the objective function $\mathbf{J}_{2}$ in (13), and for Strategy 3 calculate the objective function $\mathbf{J}_{3}$ in (16).

Step 4. Among the candidate vectors, choose the one which gives the minimum objective function; then, the problem is solved.

After the transmit beamspace matrix is solved by using the proposed randomization algorithm, the actual transmit signal can be determined according to the following relationship: $\mathbf{s}(t)=\left[s_{1}(t), \ldots, s_{M}(t)\right]^{T}=\mathbf{W}^{*} \boldsymbol{\varphi}(t)$.
3.3. Cramer-Rao Bound. The CRB for DOA estimation of unknown target represents the best performance of any unbiased estimator. As the signal model in (3) satisfies the statistical model $\mathbf{y}(\tau) \sim N_{c}\{\mu(\tau)$, Cov $\}$, where $N_{c}\{\cdot\}$ denotes the complex Gaussian probability density function, $\mu(\tau)$ is the mean of $\mathbf{y}(\tau)$ and Cov is its covariance matrix. The CRB on estimating DOAs is derived by assuming $\mu(\tau)=\mathbf{0}$ and $\operatorname{Cov}=E\left\{\mathbf{y}(\tau) \mathbf{y}(\tau)^{H}\right\}=\mathbf{V S V}^{H}+\sigma_{n}^{2} \mathbf{I}_{K N}$. Note that the signal model has the same form as the signal model used in [20] to derive the CRB for DOA estimation in conventional array processing. Therefore, the CRB matrix for DOA estimation with transmit beamspace matrix can be calculated as follows:

$$
\operatorname{CRB}(\boldsymbol{\theta})=\frac{\sigma_{z}^{2} K}{2 T P_{t}}\left\{\operatorname{Re}\left(\mathbf{D}^{H} \mathbf{P}_{\mathbf{V}}^{\perp} \mathbf{D} \odot \mathbf{G}^{T}\right)\right\}^{-1},
$$

where $\odot$ denotes the Hadamard product, $T$ is the number of snapshots, $P_{t}$ is the total transmit energy, and $\mathbf{P}_{\mathbf{V}}^{\perp}=$ $\mathbf{I}_{K N}-\mathbf{V}\left(\mathbf{V}^{H} \mathbf{V}\right)^{-1} \mathbf{V}^{H}$ is the projection matrix onto the space spanned by the columns of $\mathbf{V} . \mathbf{V}=\left[\left(\mathbf{W}^{H} \mathbf{a}\left(\theta_{1}\right)\right) \otimes\right.$ $\left.\mathbf{b}\left(\theta_{1}\right), \ldots,\left(\mathbf{W}^{H} \mathbf{a}\left(\theta_{P}\right)\right) \otimes \mathbf{b}\left(\theta_{P}\right)\right]$ represents the virtual steering vector, $\mathbf{G}=\mathbf{S V}^{H} \mathbf{R}^{-1} \mathbf{V S}$, where $\mathbf{R}$ stands for the covariance matrix of the received signal and $\mathbf{S}$ is the covariance matrix of reflection coefficients. $\mathbf{D}=\left[\mathbf{d}\left(\theta_{1}\right), \ldots, \mathbf{d}\left(\theta_{P}\right)\right]$ is the matrix whose $l$ th column is given by the derivative of the $l$ th column of $\mathbf{V}$ with respect to $\theta_{l}$; that is,

$$
\begin{aligned}
\mathbf{d}\left(\theta_{l}\right) & =\frac{\partial \mathbf{v}\left(\theta_{l}\right)}{\partial \theta_{l}}=\frac{\partial\left(\mathbf{W}^{H} \mathbf{a}\left(\theta_{l}\right)\right) \otimes \mathbf{b}\left(\theta_{l}\right)}{\partial \theta_{l}} \\
& =\mathbf{W}^{H} \mathbf{a}^{\prime}\left(\theta_{l}\right) \otimes \mathbf{b}\left(\theta_{l}\right)+\mathbf{W}^{H} \mathbf{a}\left(\theta_{l}\right) \otimes \mathbf{b}^{\prime}\left(\theta_{l}\right),
\end{aligned}
$$

where $\mathbf{a}^{\prime}\left(\theta_{l}\right)=\partial \mathbf{a}\left(\theta_{l}\right) / \partial \theta_{l}$ and $\mathbf{b}^{\prime}\left(\theta_{l}\right)=\partial \mathbf{b}\left(\theta_{l}\right) / \partial \theta_{l}$. It is worth noting that three proposed sidelobe suppression strategies lead to different transmit beamspace matrices, which result in DOA estimation performance divergence. A comprehensive comparison between the aforementioned strategies is taken in the following section.

Note that the complexity of eigendecomposition based DOA estimation techniques, such as ESPRIT, will increase with the numbers of the transmitted orthogonal waveforms $K$ and the receive array antennas $N$. The proposed model can transmit fewer orthogonal waveforms by introducing transmit beamspace matrix with the DOA estimation computational complexity of $O\left(K^{3} N^{3}\right)$. On the other hand, the DOA estimation computational complexity of traditional MIMO radar is $O\left(M^{3} N^{3}\right)$, where $M \geq K$ and $M$ is the number of transmit array antennas. Therefore, the proposed method enjoys a lower DOA estimation computational complexity compared with traditional MIMO radar.

\section{Simulation Results}

In this section, to validate the performance of the proposed sidelobe suppression strategies, some numerical examples are presented. In all of the following simulations, we assume a uniform linear transmit array of $M=10$ antennas spaced half a wavelength apart and a nonuniform linear receive array 


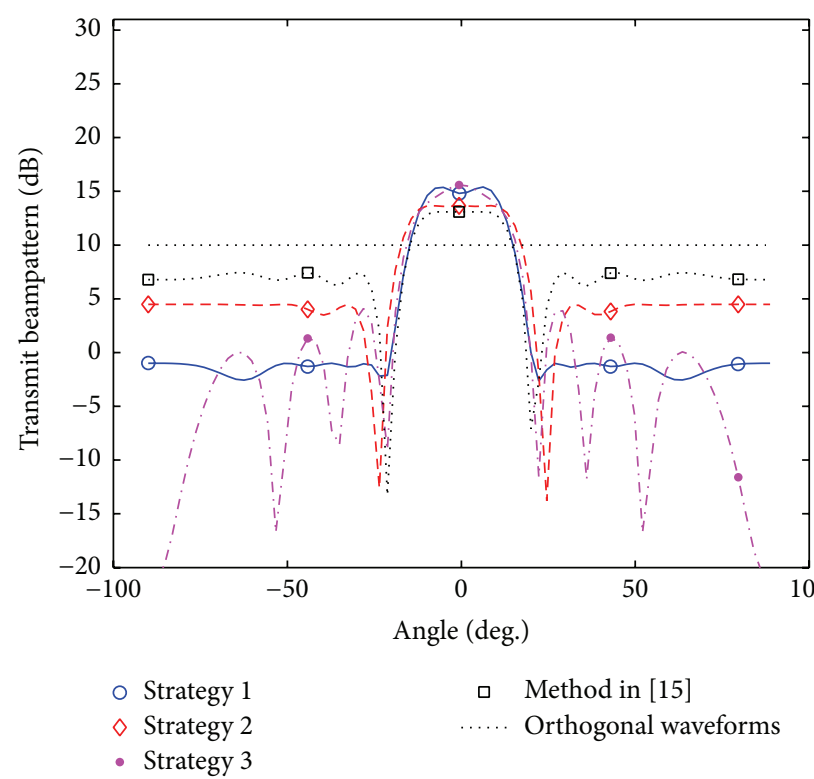

FIGURE 1: Transmit beampatterns of the orthogonal waveforms, method in [15], and the proposed strategies in Example 1.

of $N=10$ elements. The locations of the receive antennas are randomly drawn from the set $[0,9]$ measured in half a wavelength. Noise signals are assumed to be Gaussian, zero-mean, and white both temporally and spatially. In each example, targets are assumed to lie within a given spatial sector, the total transmit energy $P_{t}=M$, and the orthogonal waveforms number $K=2$. The proposed methods are compared with traditional MIMO radar and the waveforms designed in [15], and the ESPRIT algorithm is used for DOA estimation for all tested methods. The orthogonal baseband waveforms used for the traditional MIMO radar are $\varphi_{k}(t)=\sqrt{1 / T_{p}} e^{j 2 \pi m t / T_{p}},(m=1, \ldots, M)$, where $T_{p}$ is the pulse duration. For the proposed method, the first $K=$ 2 orthogonal waveforms are employed as the base signal. Throughout all simulations, the number of radar pulses used is 50 and the number of samples within one radar pulse is 1024. The DOA estimation simulation results are calculated based on 500 independent Monte Carlo runs.

Example 1 (transmit energy focusing on a single sector). In this example, the sector of interest $\Theta=\left[-10^{\circ}, 10^{\circ}\right]$ is taken, and the out-of-sector region is taken as $\bar{\Theta}=\left[-90^{\circ},-20^{\circ}\right] \cup$ $\left[20^{\circ}, 90^{\circ}\right]$. The number of uniform sampling points in sector $\Theta$ and out-of-sector $\bar{\Theta}$ are set as $L=100$ and $S=400$, respectively. In Strategy 1, the sidelobe threshold is set as an empirical value $\eta_{s}=0.01$. The upper bound of the error between desired beampattern and the actual one in Strategy 2 is also set as $\xi=0.01$. Figure 1 shows the transmit beampatterns of the traditional MIMO radar, the method of [15], and the proposed sidelobe suppression methods. It can be seen from this figure that the power distribution of orthogonal waveforms is uniform within the desired sector. The method of [15] provides concentration of power in the desired sector with high sidelobes. For the proposed method, Strategy 1 has the lowest sidelobes but results in relatively large ripples in the main-lobe region. Strategy 2 has minimum main-lobe ripples but the beampattern sidelobes are higher than Strategies 1 and 3. Note that Strategy 2 is more suitable to the scenario where tough ripple control within the main-lobe region is required. Strategy 3 shows a peak appearance in the main-lobe region and scrolls down in the sidelobe regions. It can be seen that the sidelobe suppression ability of Strategy 3 lays between Strategies 1 and 2 .

To verify the DOA estimation performance of the proposed method, two targets are assumed to be located at $\theta_{1}=-5^{\circ}$ and $\theta_{2}=5^{\circ}$. Figure 2 shows the RMSEs and CRBs versus SNR for all the methods tested. Here, the RMSE of DOA estimation is defined as RMSE = $\sqrt{(1 / 2 Q) \cdot \sum_{q=1}^{Q}\left[\left(\theta_{1}-\widehat{\theta}_{1, q}\right)^{2}+\left(\theta_{2}-\widehat{\theta}_{2, q}\right)^{2}\right]}$, where $Q$ is the number of Monte Carlo trials and $\widehat{\theta}_{l, q}$ is the angle estimate of $\theta_{l}(l=1,2)$ at the $q$ th trial. As we can see from the figure, the beampattern synthesized exploiting orthogonal waveforms provides the worst CRB and RMSE performances as compared to other methods. The reason for this is that partial transmit energy is wasted in undesired sector, resulting in low SNR at the receiver. Because transmit power is focused in the desired sector, the DOA estimation performance of the method of [15] is better than orthogonal waveforms. For the proposed method, all of three sidelobe suppression strategies exhibit DOA estimation performance improvement compared to [15]. It can be observed from the figure that the performances of the three proposed strategies are near. Specifically, Strategy 1 has the best CRB and RMSE performances, which can be attributed to the fact that Strategy 1 has the lowest sidelobes, and the DOA estimation performance of Strategy 3 is slightly better than Strategy 2.

To assess the proposed method's ability to resolve closely located targets, we move two targets to the locations $\theta_{1}=$ $7^{\circ}$ and $\theta_{2}=8^{\circ}$. The performance of all methods tested is given in terms of the probability of target resolution. Note that the targets are considered to be resolved if the condition $\left|\widehat{\theta}_{l}-\theta_{l}\right| \leq \Delta \theta / 2, l=1,2$ is satisfied, where $\Delta \theta=\left|\theta_{2}-\theta_{1}\right|$ and $\widehat{\theta}_{l}$ denotes the estimation of $\theta_{l}$. The probability of source resolution versus SNR for all methods tested is shown in Figure 3. It can be seen from the figure that traditional MIMO radar exploiting orthogonal waveforms have the worst probability of target resolution because of the fact that it does not incorporate any type of transmit energy focusing technique. For the proposed method, all of the three sidelobe suppression strategies exhibit better target resolution performance than traditional MIMO radar and the method of [15]. Specifically, Strategy 1 has the best probability of target resolution performance, and Strategy 3 is slightly better than Strategy 2. It can be indicated that transmit beampattern with low sidelobe levels achieves better performance of target resolution.

Example 2 (transmit energy focusing on multiple sectors). In this example, two targets are assumed to lie within two spatial sectors, which are taken as $\Theta=\left[-40^{\circ},-20^{\circ}\right] \cup\left[20^{\circ}, 40^{\circ}\right]$. The out-of-sector regions are taken as $\bar{\Theta}=\left[-90^{\circ}, 50^{\circ}\right] \cup$ $\left[-10^{\circ}, 10^{\circ}\right] \cup\left[50^{\circ}, 90^{\circ}\right]$. The number of uniform sampling 


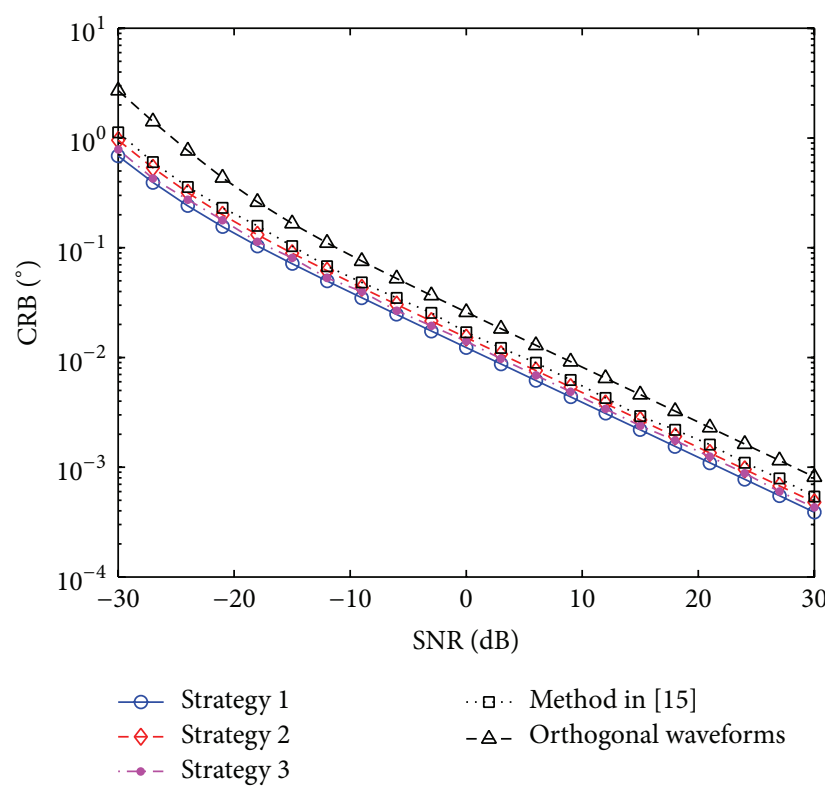

(a)

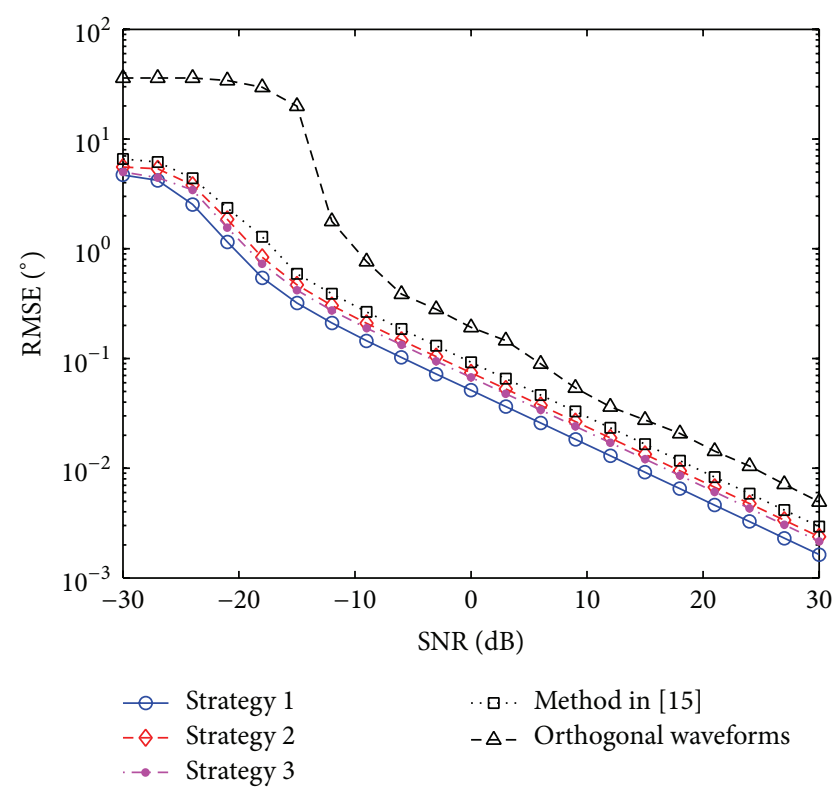

(b)

Figure 2: (a) CRB versus SNR in Example 1. (b) RMSE versus SNR in Example 1.

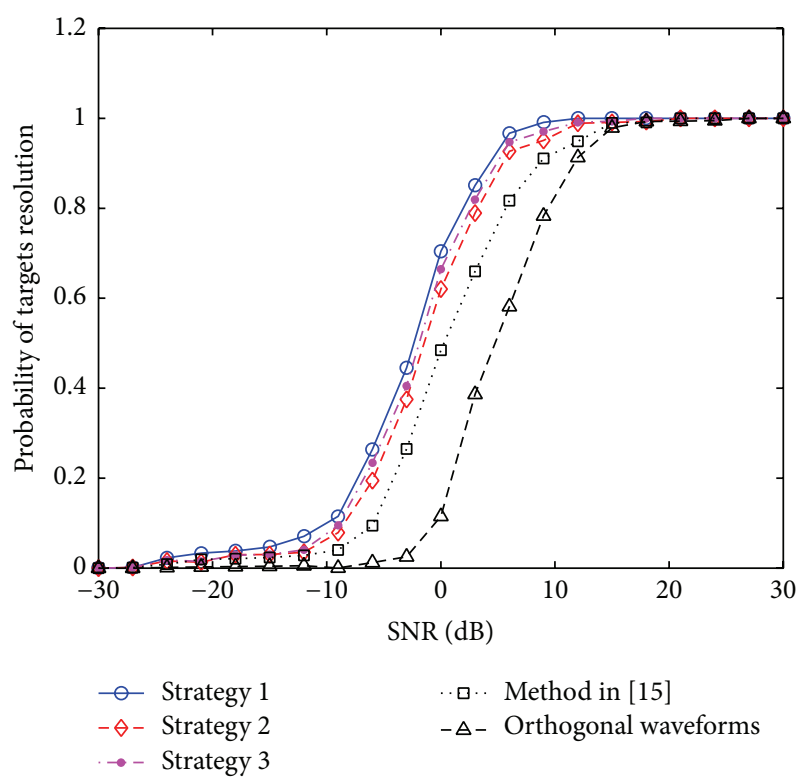

FIgURE 3: Probability of target resolution versus SNR in Example 1.

points in sector $\Theta$ and out-of-sector $\bar{\Theta}$ are set as $L=200$ and $S=300$, respectively. Figure 4 shows that the transmit beampattern synthesized exploiting orthogonal waveforms has uniform transmit power distribution. It can be seen from the figure that the method of [15] provides concentration of power in the two desired sectors with high sidelobe levels. Strategy 1 provides a transmit beampattern with lowest sidelobes but has relatively larger ripples in both desired sectors. Strategy 2 obtains the most even concentration of power in the desired sectors; however, the beampattern sidelobes of Strategy 2 are higher than Strategies 1 and 3. The

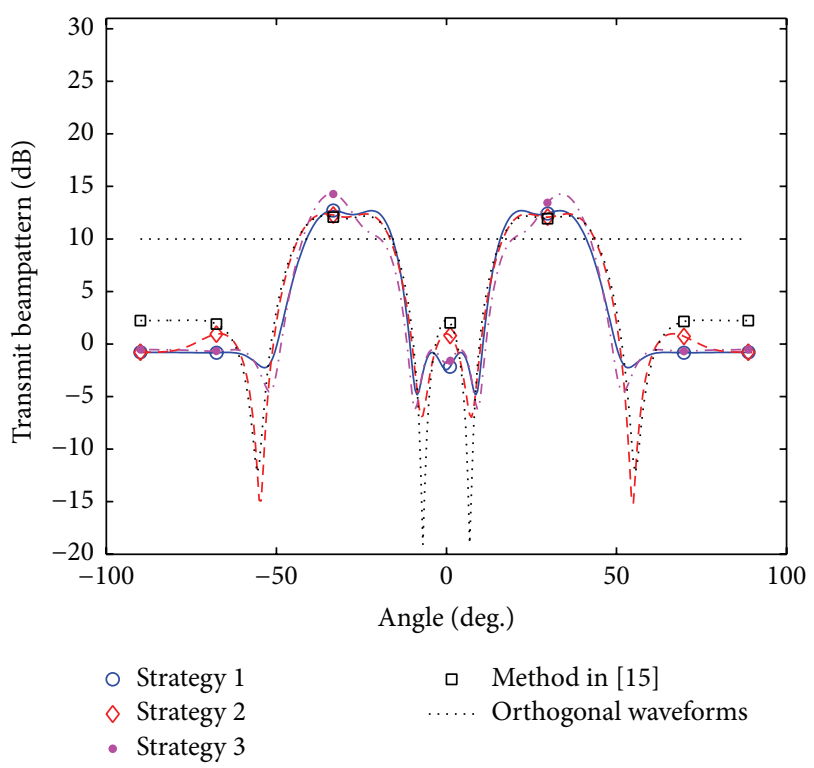

FIgURE 4: Transmit beampatterns of the orthogonal waveforms, method in [15], and the proposed strategies in Example 2.

sidelobe suppression ability of Strategy 3 still lays between Strategies 1 and 2 .

Figure 5 shows the RMSEs and CRBs versus SNR for all the methods tested when the targets are assumed to be located at $\theta_{1}=-30^{\circ}$ and $\theta_{2}=25^{\circ}$ with two desired sectors. It can be seen from the figure that the DOA estimation performance of the method of [15] is better than traditional MIMO radar that exploits orthogonal waveforms as expected. The proposed methods outperform the method of [15]; that is, Strategy 1 has the best CRB and RMSE performances, and the DOA estimation performance of Strategy 3 is slightly better than Strategy 


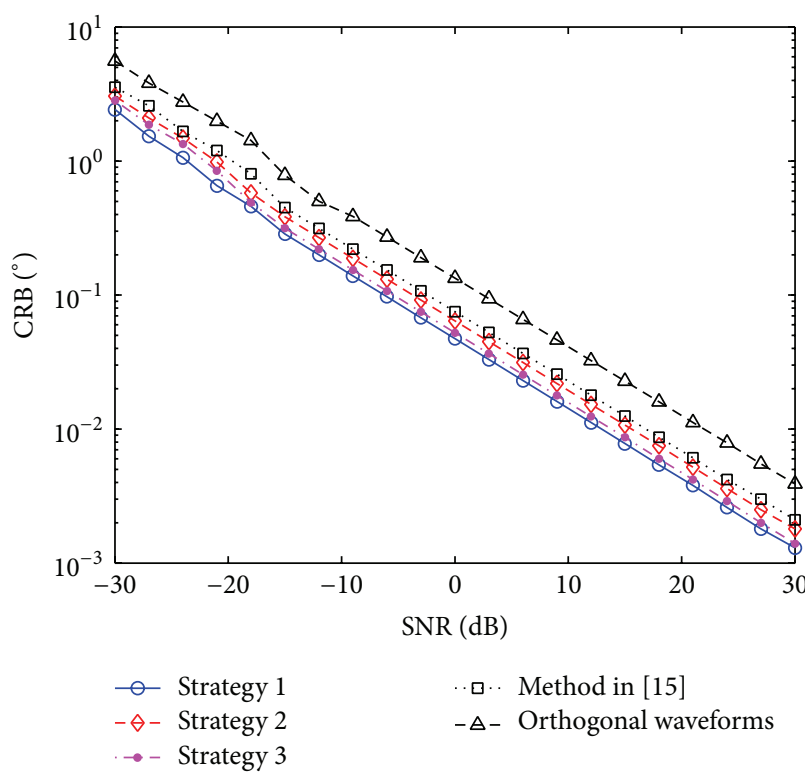

(a)

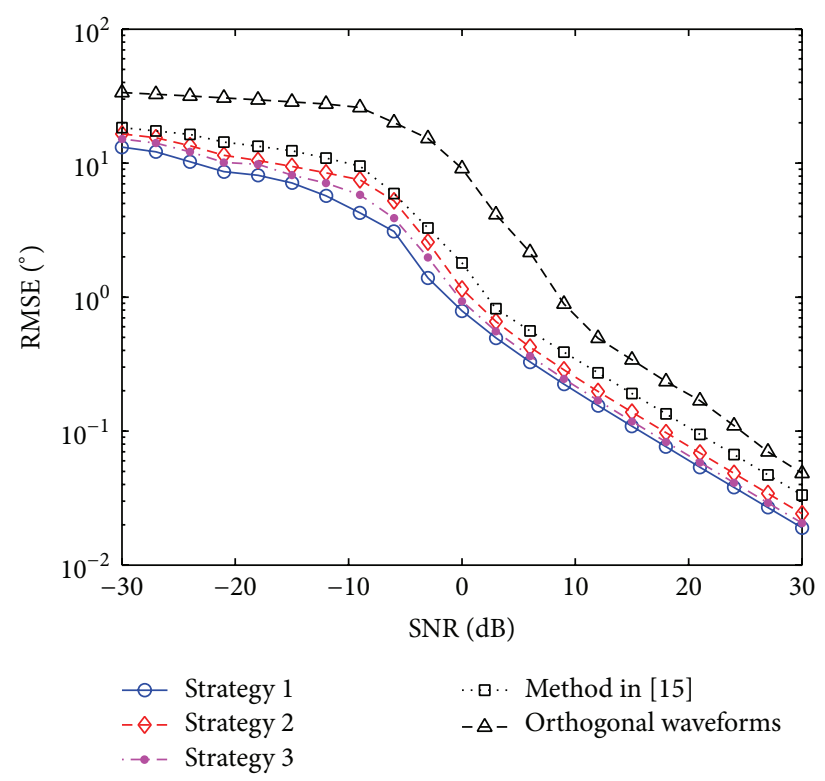

(b)

FIgURE 5: (a) CRB versus SNR in Example 2. (b) RMSE versus SNR in Example 2.

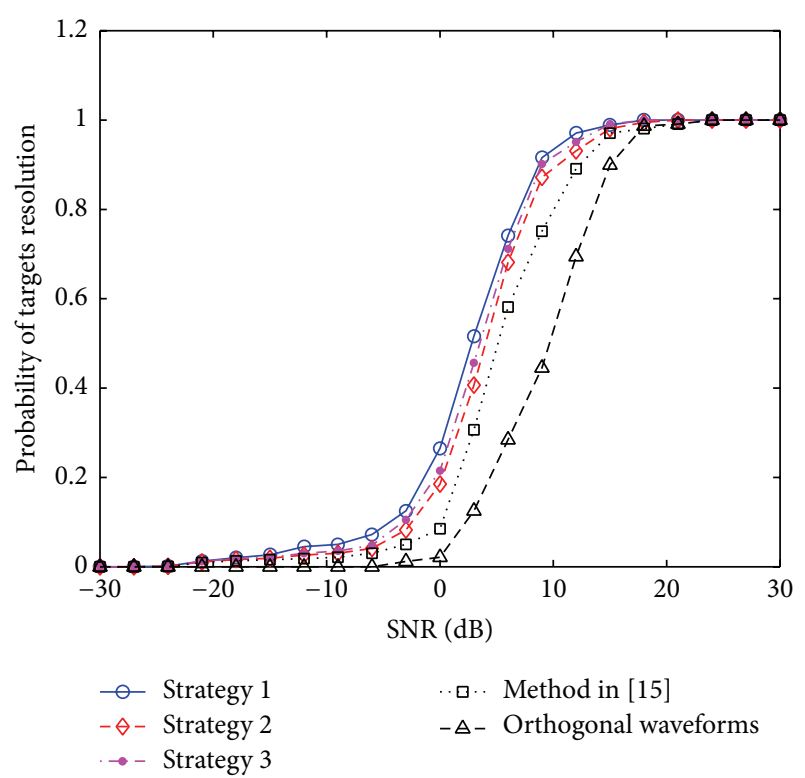

FIGURE 6: Probability of target resolution versus SNR in Example 2.

2. This confirms again the observation from the previous example that proposed sidelobe suppression strategies are effective. Moreover, by comparing the results in Figures 2 and 5 , it can be concluded that the DOA estimation performance with two desired sectors is poorer than one single desired sector case as the width of each sector is the same. The reason for this is that two-desired-sector case suffers the transmit energy dispersion compared with the single one case.

Figure 6 shows the probability of target resolution for all aforementioned methods after locating both targets at the locations $\theta_{1}=21^{\circ}$ and $\theta_{2}=22^{\circ}$. The result is analogous to the single desired sector example. In a similar way, by comparing
Figures 3 and 6, we can observe that the target resolution performance of the single desired sector case is superior to the two-desired-sector case as maintaining the width of each sector invariant.

\section{Conclusion}

The problem of MIMO radar transmit beamspace design with sidelobe suppression for DOA estimation application has been considered. Three sidelobe suppression strategies for designing the transmit beamspace matrix that enable the use of ESPRIT estimation technique have been introduced. Thus, it allows achieving a better estimation performance at lower computational cost. Semidefinite relaxation technique is used to recast the optimization problems as convex ones. Then, a randomization algorithm is proposed to extract the solutions of the original problems. CRB expression as functions of the transmit beamspace weight matrix is given. Simulation results have demonstrated the improvement in the DOA estimation performance offered by using the proposed methods as compared to the existing techniques.

\section{Conflict of Interests}

The authors declared that they do not have any commercial or associative interest that represents a conflict of interests in connection with this work.

\section{Acknowledgments}

This work is supported in part by the Important National Science and Technology Specific Project (2011ZX03003-003$02)$ and the National High Technology Research and Development Program of China (Program 863) (2012AA01A502, 2012AA01A505). 


\section{References}

[1] J. Li and P. Stoica, MIMO Radar Signal Processing, John Wiley \& Sons, Hoboken, NJ, USA, 2008.

[2] A. M. Haimovich, R. S. Blum, and L. J. Cimini, "MIMO radar with widely separated antennas," IEEE Signal Processing Magazine, vol. 25, no. 1, pp. 116-129, 2008.

[3] A. De Maio, M. Lops, and L. Venturino, "Diversity-integration tradeoffs in MIMO detection," IEEE Transactions on Signal Processing, vol. 56, no. 10, pp. 5051-5061, 2008.

[4] A. Hassanien, S. A. Vorobyov, and A. B. Gershman, "Moving target parameters estimation in non-coherent MIMO radar systems," IEEE Transactions on Signal Processing, vol. 60, no. 5, pp. 2354-2361, 2012.

[5] J. Li and P. Stoica, "MIMO radar with colocated antennas," IEEE Signal Processing Magazine, vol. 24, no. 5, pp. 106-114, 2007.

[6] D. Xiaofang, C. Baixiao, Y. Minglei, and Z. Guimei, "Beamspace unitary ESPRIT algorithm for angle estimation in bistatic MIMO radar," International Journal of Antennas and Propagation, vol. 2015, Article ID 621358, 9 pages, 2015.

[7] A. Hassanien and S. A. Vorobyov, "Phased-MIMO radar: a tradeoff between phased-array and MIMO radars," IEEE Transactions on Signal Processing, vol. 58, no. 6, pp. 3137-3151, 2010.

[8] D. Wilcox and M. Sellathurai, "On MIMO radar subarrayed transmit beamforming," IEEE Transactions on Signal Processing, vol. 60, no. 4, pp. 2076-2081, 2012.

[9] D. R. Fuhrmann and G. San Antonio, "Transmit beamforming for MIMO radar systems using signal cross-correlation," IEEE Transactions on Aerospace and Electronic Systems, vol. 44, no. 1, pp. 171-186, 2008.

[10] S. Ahmed, J. S. Thompson, Y. R. Petillot, and B. Mulgrew, "Unconstrained synthesis of covariance matrix for MIMO radar transmit beampattern," IEEE Transactions on Signal Processing, vol. 59, no. 8, pp. 3837-3849, 2011.

[11] P. Stoica, J. Li, and X. Zhu, "Waveform synthesis for diversitybased transmit beampattern design," IEEE Transactions on Signal Processing, vol. 56, no. 6, pp. 2593-2598, 2008.

[12] S. Ahmed, J. S. Thompson, Y. R. Petillot, and B. Mulgrew, "Finite alphabet constant-envelope waveform design for MIMO radar," IEEE Transactions on Signal Processing, vol. 59, no. 11, pp. 53265337, 2011

[13] A. Hassanien and S. A. Vorobyov, "Transmit energy focusing for DOA estimation in MIMO radar with colocated antennas," IEEE Transactions on Signal Processing, vol. 59, no. 6, pp. 26692682, 2011.

[14] S. Ahmed and M.-S. Alouini, "MIMO radar transmit beampattern design without synthesising the covariance matrix," IEEE Transactions on Signal Processing, vol. 62, no. 9, pp. 2278-2289, 2014.

[15] A. Khabbazibasmenj, A. Hassanien, S. A. Vorobyov, and M. W. Morency, "Efficient transmit beamspace design for search-free based DOA estimation in MIMO radar," IEEE Transactions on Signal Processing, vol. 62, no. 6, pp. 1490-1500, 2014.

[16] Z.-Q. Luo, W.-K. Ma, A. C. So, Y. Ye, and S. Zhang, "Semidefinite relaxation of quadratic optimization problems," IEEE Signal Processing Magazine, vol. 27, no. 3, pp. 20-34, 2010.

[17] M. Grant and S. Boyd, "CVX: matlab software for disciplined convex programming: CVX version 1.21,” 2011, http://cvxr.com/ cvx.
[18] P. Stoica, J. Li, and Y. Xie, "On probing signal design for MIMO radar," IEEE Transactions on Signal Processing, vol. 55, no. 8, pp. 4151-4161, 2007.

[19] S. Boyd and L. Vandenberghe, Convex Optimization, Cambridge University Press, Cambridge, UK, 2004.

[20] P. Stoica, E. G. Larsson, and A. B. Gershman, "The stochastic CRB for array processing: a textbook derivation," IEEE Signal Processing Letters, vol. 8, no. 5, pp. 148-150, 2001. 


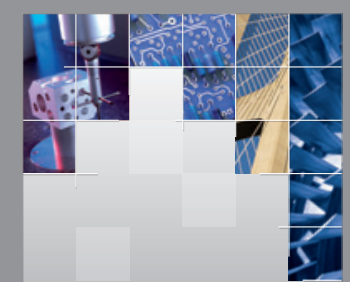

\section{Enfincering}
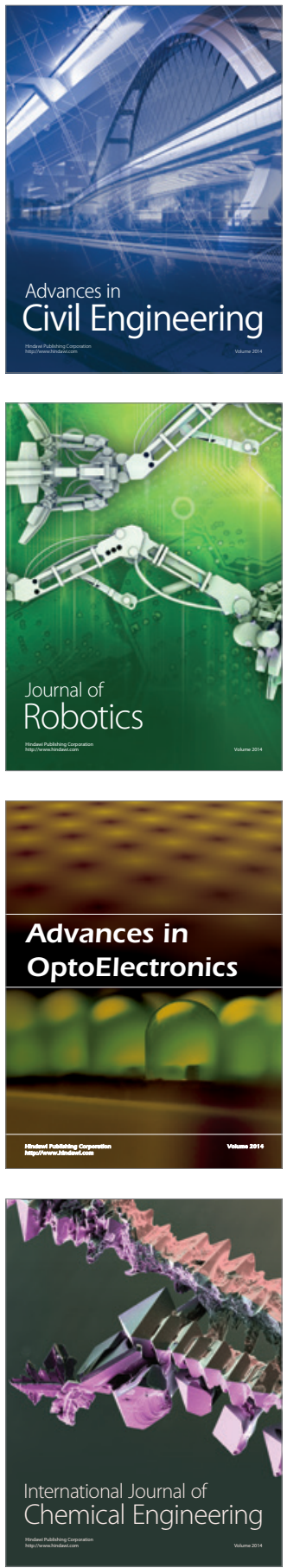

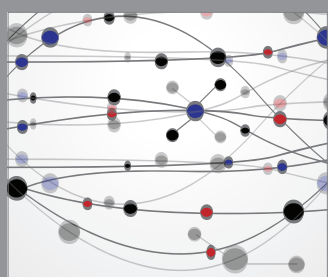

The Scientific World Journal

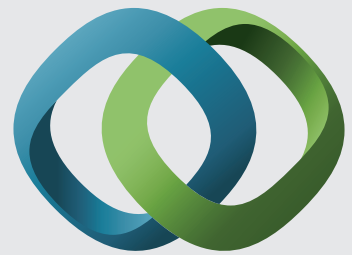

\section{Hindawi}

Submit your manuscripts at

http://www.hindawi.com
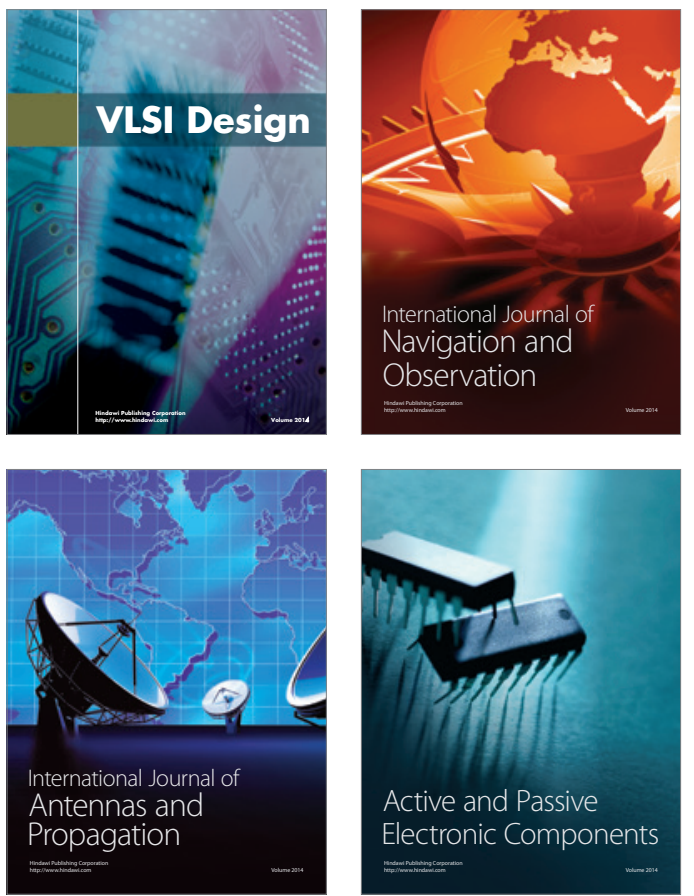
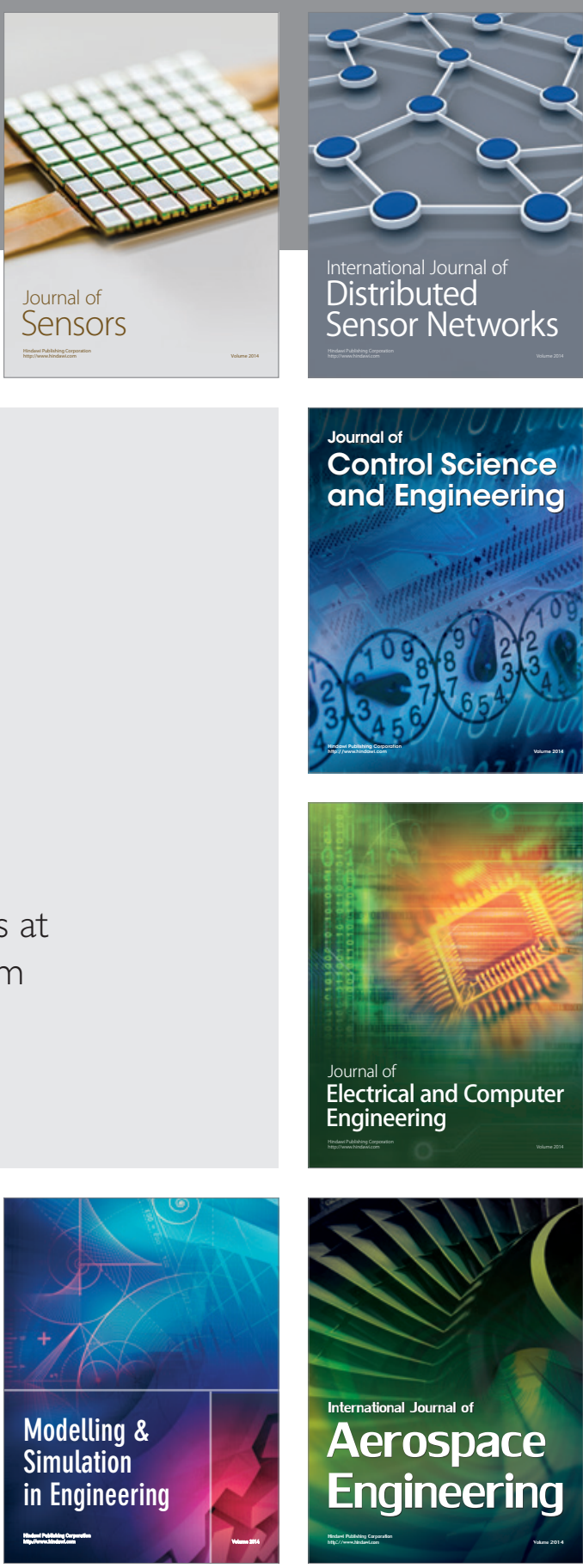

International Journal of

Distributed

Sensor Networks

Journal of

Control Science

and Engineering
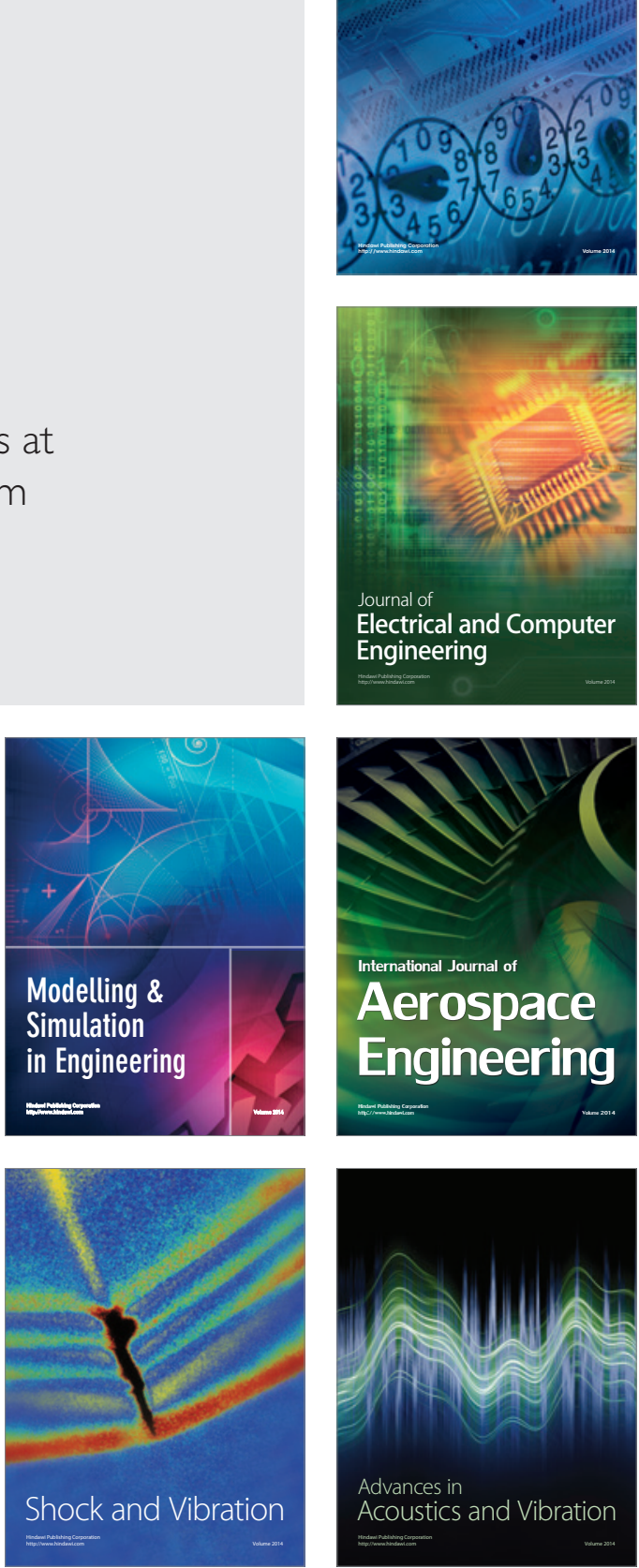